\title{
First eigenvalue of Schrödinger operator of space-like hypersurfaces
}

\author{
Shichang Shu ${ }^{1} \quad$ Sanyang Liu $^{2}$
}

(Received 12 November 2008; revised 13 April 2010)

\begin{abstract}
We introduce two Schrödinger operators of compact space-like hypersurfaces in a de Sitter space. If the hypersurfaces have constant mean curvature or constant scalar curvature, we obtain some spectral characterisations of totally umbilical space-like hypersurfaces by the first eigenvalue of the Schrödinger operators.
\end{abstract}

\section{Contents}

1 Introduction

E84

2 Preliminaries

E86

3 Proof of theorems

E89

References

E94

http://anziamj . austms.org . au/ojs/index.php/ANZIAMJ/article/view/1618 gives this article, (c) Austral. Mathematical Soc. 2010. Published April 18, 2010. ISSN 1446-8735. (Print two pages per sheet of paper.) 


\section{Introduction}

Space-like hypersurfaces with constant mean curvature or constant scalar curvature in an arbitrary Lorentz manifold play an important role in general relativity. Space-like hypersurfaces in a Lorentz manifold have recently been investigated by many researchers from both physical and mathematical points of view. Let $M_{1}^{n+1}(c)$ be an $(n+1)$-dimensional Lorentzian space form with constant sectional curvature $c$. According to $c>0, c=0$ or $c<0$, it is called a de Sitter space, a Minkowski space or an anti-de Sitter space, respectively, and is denoted by $S_{1}^{\mathfrak{n}+1}(\mathrm{c}), \mathrm{R}_{1}^{\mathrm{n}+1}$ or $\mathrm{H}_{1}^{\mathrm{n}+1}(\mathrm{c})$. When $\mathrm{c}=1$, we denote the de Sitter space by $S_{1}^{n+1}$. A hypersurface in a Lorentzian manifold is said to be space-like if the induced metric on the hypersurface is positive definite.

We know that hypersurfaces with constant mean curvature in a Riemannian manifold $\mathrm{M}^{\mathrm{n}+1}(\mathrm{c})$ of constant sectional curvature $\mathrm{c}$ are critical points of the area functional under variations that keep constant a certain volume function. Barbosa, do Carmo and Eschenburg [4] studied the stability for hypersurfaces of constant mean curvature in Riemannian manifold. In analogy with the case of constant mean curvature, questions of stability can be considered for hypersurfaces with constant scalar curvature. Alencar, do Carmo and Colares [2] extended the study of stability to hypersurfaces with constant scalar curvature. As researched by $\mathrm{C}$. Wu [16] for minimal submanifolds in a unit sphere, Alías et al. [3] and Cheng [9] studied the first eigenvalue of some Jacobi operator of hypersurfaces with constant mean curvature or constant scalar curvature in a unit sphere and obtained some spectral characterizations of so called $\mathrm{H}(\mathrm{r})$-torus $S^{\mathrm{n}-1}(\mathrm{r}) \times \mathrm{S}^{1}\left(\sqrt{1-\mathrm{r}^{2}}\right)$ or Riemannian product $S^{\mathrm{m}}(\mathrm{r}) \times$ $S^{n-m}\left(\sqrt{1-r^{2}}\right), 1 \leqslant m \leqslant n-1$.

Comparing the stability for hypersurfaces with constant mean curvature or constant scalar curvature in Riemannian manifolds, Barbosa, Oliker [5], Liu and Deng [12] studied the stability for space-like hypersurfaces with constant mean curvature or constant scalar curvature in Lorentz manifolds. From the 
results of Barbosa and Oliker [5, 6], we know that constant mean curvature space-like hypersurfaces are solutions to a variational problem. They are critical points of the area functional for variations that leave constant a certain volume function.

We define two Schrödinger operators $L_{H}$ and $L_{R}$ by (1) and (2) and obtain some spectral characterizations of totally umbilical space-like hypersurfaces by the first eigenvalue of the Schrödinger operator $L_{H}$ or $L_{R}$.

Since space-like hypersurfaces have particular structure, from the definition of the Schrödinger operator $L_{H}$ or $L_{R}$, we notice that the Schrödinger operators $\mathrm{L}_{H}$ and $\mathrm{L}_{R}$ are different from that of the Riemannian hypersurface which were studied by Alías, Barros and Brasil [3] and Cheng [9]. In the case of space-like hypersurfaces, the first eigenvalues of $L_{H}$ and $L_{R}$ have special forms.

In a neighbourhood of a point $x$ of the space-like hypersurface $M$, we choose an orthonormal frame field $\left\{e_{1}, \ldots, e_{n}\right\}$ such that $h_{i j}=\lambda_{i} \delta_{i j}$ at $x$, where $h_{i j}$ are the components of the second fundamental form of $M$. Let $H$ denote the mean curvature of $M$. We introduce the operator $\phi$ by

$$
\langle\phi X, Y\rangle=\langle h X, Y\rangle-H\langle X, Y\rangle
$$

Putting $\phi=\sum_{i, j} \phi_{i j} \omega_{i} \otimes \omega_{j}$, where $\phi_{i j}=h_{i j}-H \delta_{i j}$, we can see that $\phi$ is traceless, that the basis $\left\{e_{1}, \ldots, e_{n}\right\}$ also diagonalizes $\phi$ at $\chi$ with eigenvalues $\mu_{i}=\lambda_{i}-H$, and that

$$
|\phi|^{2}=\sum_{i} \mu_{i}^{2}=\frac{1}{2 n} \sum_{i, j}\left(\lambda_{i}-\lambda_{j}\right)^{2}=S-n H^{2},
$$

where $S$ denotes the norm square of the second fundamental form of $M$. We know that $|\phi|^{2} \equiv 0$ if and only if $M$ is totally umbilical.

Before announcing our main results, we introduce the following Schrödinger operators:

$$
\mathrm{L}_{\mathrm{H}}=-\Delta+|\phi|^{2}-\frac{\mathrm{n}(\mathrm{n}-2)}{\sqrt{\mathrm{n}(\mathrm{n}-1)}} \mathrm{H}|\phi|,
$$




$$
\mathrm{L}_{\mathrm{R}}=-\square+\frac{1}{\mathrm{nH}}|\phi|^{4}+\frac{1-\mathrm{H}^{2}}{\mathrm{H}}|\phi|^{2},
$$

where the differential operator

$$
\square f=\sum_{i, j=1}^{n}\left(n H \delta_{i j}-h_{i j}\right) f_{i j},
$$

for any $C^{2}$-function $f$, which was introduced and used by S. Y. Cheng and Yau [11]. Now we state our results.

Theorem 1 Let $\mathrm{M}$ be an $\mathrm{n}$-dimensional compact orientable space-like hypersurface in an $(n+1)$-dimensional de Sitter space $\mathrm{S}_{1}^{\mathfrak{n}+1}$ with constant mean curvature $\mathrm{H}$. Denote by $\lambda_{1}^{\mathrm{L}_{\mathrm{H}}}$ the first eigenvalue of the Schrödinger operator $\mathrm{L}_{\mathrm{H}}$. If $\lambda_{1}^{\mathrm{L}_{\mathrm{H}}} \geqslant-\mathrm{n}\left(1-\mathrm{H}^{2}\right)$, then $\mathrm{M}$ is totally umbilical.

Theorem 2 Let $\mathrm{M}$ be an $\mathrm{n}$-dimensional compact orientable space-like hypersurface in an $(n+1)$-dimensional de Sitter space $\mathrm{S}_{1}^{\mathrm{n}+1}$ with constant scalar curvature $\mathrm{n}(\mathrm{n}-1) \mathrm{R}(\mathrm{R}<1)$. Denote by $\lambda_{1}^{\mathrm{L}_{\mathrm{R}}}$ the first eigenvalue of the Schrödinger operator $\mathrm{L}_{\mathrm{R}}$. Then

$$
\lambda_{1}^{L_{R}} \leqslant \frac{n-2}{\sqrt{n(n-1)}} \max |\phi|^{3} \quad \text { and } \quad \lambda_{1}^{L_{R}}=\frac{n-2}{\sqrt{n(n-1)}} \max |\phi|^{3},
$$

if and only if $\mathrm{M}$ is totally umbilical.

\section{Preliminaries}

Let $M$ be an $n$-dimensional space-like hypersurface in an $(n+1)$-dimensional de Sitter space $S_{1}^{\mathfrak{n}+1}$. We choose a local field of semi-Riemannian orthonormal frames $\left\{e_{1}, \ldots, e_{n+1}\right\}$ in $S_{1}^{n+1}$ such that at each point of $M,\left\{e_{1}, \ldots, e_{n}\right\}$ span the tangent space of $M$ and form an orthonormal frame there. We use the following convention on the range of indices:

$$
1 \leqslant A, B, C, \ldots \leqslant n+1 ; \quad 1 \leqslant i, j, k, \ldots \leqslant n .
$$


Let $\left\{\omega_{1}, \ldots, \omega_{n+1}\right\}$ be the dual frame field so that the semi-Riemannian metric of the de Sitter space $S_{1}^{n+1}$ is $d \bar{s}^{2}=\sum_{i} \omega_{i}^{2}-\omega_{n+1}^{2}=\sum_{A} \epsilon_{A} \omega_{A}^{2}$, where $\epsilon_{i}=1$ and $\epsilon_{n+1}=-1$.

Restrict to $M$,

$$
\omega_{n+1}=0 .
$$

Cartan's Lemma implies that

$$
\omega_{n+1 i}=\sum_{j} h_{i j} \omega_{j}, \quad h_{i j}=h_{j i} .
$$

The Gauss equation is

$$
R_{i j k l}=\left(\delta_{i k} \delta_{j l}-\delta_{i l} \delta_{j k}\right)-\left(h_{i k} h_{j l}-h_{i l} h_{j k}\right),
$$

where $R_{i j k l}$ are the components of the curvature tensor of $M$ and

$$
h=\sum_{i, j} h_{i j} \omega_{i} \otimes \omega_{j}
$$

is the second fundamental form of $M$. From the above equation,

$$
n(n-1)(R-1)=S-n^{2} H^{2},
$$

where $n(n-1) R$ is the scalar curvature of $M, H$ is the mean curvature and $S=\sum_{i, j} h_{i j}^{2}$ is the norm square of the second fundamental form of $M$.

The Codazzi equation is

$$
h_{i j k}=h_{i k j} .
$$

We consider the differential operator $\square$ defined by

$$
\square \mathrm{f}=\sum_{i, j=1}\left(n H \delta_{i j}-h_{i j}\right) f_{i j},
$$

where $d f=\sum_{i} f_{i} \omega_{i}, \sum_{i, j} f_{i j} \omega_{j}=d f_{i}+\sum_{j} f_{j} \omega_{j i}$. 
We know that the Laplace-Beltrami operator $\Delta$ is always elliptic. From the discussion of Cheng and Yau [11], we know that the operator $\square$ is selfadjoint, and from the result of Cheng and Ishikawa [10], we know that if $M$ is an $n$-dimensional space-like hypersurface in $S_{1}^{\mathfrak{n}+1}$ with constant scalar curvature $n(n-1) R$ and $R<1$, then $\square$ is an elliptic operator (see a Lemma of Cheng and Ishikawa [10]). Let $\lambda_{1}^{L_{H}}$ and $\lambda_{1}^{L_{R}}$ be the first eigenvalues of the Schrödinger operators $L_{H}$ and $L_{R}$, respectively. Since $\Delta$ and $\square$ are elliptic operators, from (1) and (2) we know that $L_{H}$ and $L_{R}$ are elliptic operators. We can use the min-max characterisation of $\lambda_{1}^{L_{H}}$ and $\lambda_{1}^{L_{R}}$, as

$$
\begin{aligned}
& \lambda_{1}^{L_{H}}=\min \left\{\frac{\int_{M} f L_{H}(f) d v}{\int_{M} f^{2} d v}: f \in \mathcal{C}^{\infty}(M), f \neq \equiv 0\right\}, \\
& \lambda_{1}^{L_{R}}=\min \left\{\frac{\int_{M} f L_{R}(f) d v}{\int_{M} f^{2} d v}: f \in \mathcal{C}^{\infty}(M), f \neq \equiv 0\right\} .
\end{aligned}
$$

From the result of Brasil Jr. et al. [7], we know that if $M$ is a orientable space-like hypersurface with constant mean curvature $\mathrm{H}$ in $S_{1}^{\mathfrak{n}+1}$, then

$$
\begin{aligned}
\frac{1}{2} \Delta|\phi|^{2} & =|\nabla \phi|^{2}+\left(|\phi|^{2}\right)^{2}-n H \operatorname{tr} \phi^{3}+n\left(1-H^{2}\right)|\phi|^{2} \\
& \geqslant|\nabla \phi|^{2}+|\phi|^{2}\left\{|\phi|^{2}-\frac{n(n-2) H}{\sqrt{n(n-1)}}|\phi|+n\left(1-H^{2}\right)\right\},
\end{aligned}
$$

where the following result due to Okumura [14] and Alencar, do Carmo [1] is used:

Let $\mu_{1}, \mu_{2}, \ldots, \mu_{n}$ be real numbers such that $\sum_{i} \mu_{i}=0$, and $\sum_{i} \mu_{i}^{2}=\beta^{2}$, where $\beta=$ constant $\geqslant 0$, then

$$
-\frac{n-2}{\sqrt{n(n-1)}} \beta^{3} \leqslant \sum_{i} \mu_{i}^{3} \leqslant \frac{n-2}{\sqrt{n(n-1)}} \beta^{3},
$$

and equality holds in (13) if and only if at least $(n-1)$ of the numbers $\mu_{i}$ are equal. 
From the calculation of Liu [13] or Shu [15], we conclude that for orientable space-like hypersurfaces with constant scalar curvature $n(n-1) R$ in $S_{1}^{n+1}$,

$$
\begin{aligned}
\square(\mathrm{nH}) & =|\nabla h|^{2}-\mathrm{n}^{2}|\nabla \mathrm{H}|^{2}+\left(|\phi|^{2}\right)^{2}-n H \operatorname{tr} \phi^{3}+\mathrm{n}\left(1-\mathrm{H}^{2}\right)|\phi|^{2} \\
& \geqslant|\nabla h|^{2}-\mathrm{n}^{2}|\nabla \mathrm{H}|^{2}+|\phi|^{2}\left\{|\phi|^{2}-\frac{\mathrm{n}(\mathrm{n}-2) \mathrm{H}}{\sqrt{\mathrm{n}(\mathrm{n}-1)}}|\phi|+\mathrm{n}\left(1-\mathrm{H}^{2}\right)\right\},
\end{aligned}
$$

and if $R<1$, then

$$
|\nabla h|^{2} \geqslant \mathrm{n}^{2}|\nabla \mathrm{H}|^{2}
$$

\section{Proof of theorems}

We firstly state a proposition proved by making use of a method similar to that used by C. Wu [16] or A. A. Barros et al. [8] for a Riemannian manifold.

Proposition 3 Let $\mathrm{M}$ be an $\mathrm{n}$-dimensional space-like hypersurface in an $(\mathrm{n}+1)$-dimensional de Sitter space $\mathrm{S}_{1}^{\mathrm{n}+1}$. Then

$$
\left.\left.|\nabla| \phi\right|^{2}\right|^{2} \leqslant \frac{4 n|\phi|^{2}}{n+2}|\nabla \phi|^{2}
$$

Proof: We easily see that $\phi_{i j}=h_{i j}-H \delta_{i j}$ with eigenvalues $\mu_{i}=\lambda_{i}-H$ and $\phi$ is traceless, that is, $\sum_{k} \phi_{k k}=0$. By the Cauchy-Schwarz inequality

$$
\begin{aligned}
\left.\left.|\nabla| \phi\right|^{2}\right|^{2} & =4 \sum_{k}\left(\sum_{i, j} \phi_{i j} \phi_{i j k}\right)^{2}=4 \sum_{k}\left(\sum_{i} \mu_{i} \phi_{i i k}\right)^{2} \\
& \leqslant 4 \sum_{i} \mu_{i}^{2} \sum_{i, k}\left(\phi_{i i k}\right)^{2}=4|\phi|^{2}\left(\sum_{i}\left(\phi_{i i i}\right)^{2}+\sum_{i, k, k \neq i}\left(\phi_{i i k}\right)^{2}\right) .
\end{aligned}
$$


Since $\phi$ is traceless, $\phi_{i i i}=-\sum_{k, k \neq i} \phi_{k k i}$. Thus

$$
\sum_{i}\left(\phi_{i i i}\right)^{2}=\sum_{i}\left(\sum_{k, k \neq i} \phi_{k k i}\right)^{2} \leqslant(n-1) \sum_{k, i, k \neq i}\left(\phi_{i i k}\right)^{2} .
$$

From above two inequalities,

$$
\left.\left.|\nabla| \phi\right|^{2}\right|^{2} \leqslant 4 n|\phi|^{2} \sum_{k, i, k \neq i}\left(\phi_{i i k}\right)^{2} .
$$

From (8), we know that $\phi_{i j k}$ are symmetric for the three indices $i, j, k$. By the above inequality and (17),

$$
\begin{aligned}
\left.\left.|\nabla| \phi\right|^{2}\right|^{2} & =|\phi|^{2} \sum_{i, j, k}\left(\phi_{i j k}\right)^{2} \\
& =|\phi|^{2}\left(\sum_{i}\left(\phi_{i i i}\right)^{2}+3 \sum_{i, k, i \neq k}\left(\phi_{i i k}\right)^{2}+6 \sum_{i<j<k}\left(\phi_{i j k}\right)^{2}\right) \\
& \geqslant|\phi|^{2}\left(\sum_{i}\left(\phi_{i i i}\right)^{2}+\sum_{i, k, i \neq k}\left(\phi_{i i k}\right)^{2}+2 \sum_{i, k, i \neq k}\left(\phi_{i i k}\right)^{2}\right) \\
& \geqslant\left.\left.\frac{1}{4}|\nabla| \phi\right|^{2}\right|^{2}+\left.\left.\frac{1}{2 n}|\nabla| \phi\right|^{2}\right|^{2}=\left.\left.\frac{n+2}{4 n}|\nabla| \phi\right|^{2}\right|^{2} .
\end{aligned}
$$

This completes the proof of Proposition 3.

Proof of 1: Since $M$ is orientable, we assume that $H \geqslant 0$. For every $\varepsilon>0$, from (10), we introduce a smooth function $f_{\varepsilon}=\sqrt{\varepsilon+|\phi|^{2}}$ as the test function to estimate $\lambda_{1}^{\mathrm{L}_{H}}$. Then

$$
\Delta \mathrm{f}_{\varepsilon}=\frac{1}{2 \sqrt{\varepsilon+|\phi|^{2}}} \Delta|\phi|^{2}-\left.\left.\frac{1}{4\left(\varepsilon+|\phi|^{2}\right)^{3 / 2}}|\nabla| \phi\right|^{2}\right|^{2} .
$$


From (12) and (18) and Proposition 3,

$$
\begin{aligned}
f_{\varepsilon} \Delta f_{\varepsilon}= & \frac{1}{2} \Delta|\phi|^{2}-\left.\left.\frac{1}{4\left(\varepsilon+|\phi|^{2}\right)}|\nabla| \phi\right|^{2}\right|^{2} \\
\geqslant & |\phi|^{2}\left\{|\phi|^{2}-\frac{n(n-2) H}{\sqrt{n(n-1)}}|\phi|+n\left(1-H^{2}\right)\right\} \\
& +|\nabla \phi|^{2}-\left.\left.\frac{1}{4\left(\varepsilon+|\phi|^{2}\right)}|\nabla| \phi\right|^{2}\right|^{2} \\
\geqslant & |\phi|^{2}\left\{|\phi|^{2}-\frac{n(n-2) H}{\sqrt{n(n-1)}}|\phi|+n\left(1-H^{2}\right)\right\} \\
& +\left\{1-\frac{n|\phi|^{2}}{(n+2)\left(\varepsilon+|\phi|^{2}\right)}\right\}|\nabla \phi|^{2} .
\end{aligned}
$$

Therefore,

$$
\begin{aligned}
\mathrm{f}_{\varepsilon} \mathrm{L}_{\mathrm{H}} \mathrm{f}_{\varepsilon}= & -\mathrm{f}_{\varepsilon} \Delta \mathrm{f}_{\mathcal{\varepsilon}}+\left\{|\phi|^{2}-\frac{\mathrm{n}(\mathrm{n}-2)}{\sqrt{\mathrm{n}(\mathrm{n}-1)}} \mathrm{H}|\phi|\right\} \mathrm{f}_{\varepsilon}^{2} \\
\leqslant & -|\phi|^{2}\left\{|\phi|^{2}-\frac{\mathrm{n}(\mathrm{n}-2) \mathrm{H}}{\sqrt{\mathrm{n}(\mathrm{n}-1)}}|\phi|+n\left(1-\mathrm{H}^{2}\right)\right\} \\
& -\left\{1-\frac{n|\phi|^{2}}{(\mathrm{n}+2)\left(\varepsilon+|\phi|^{2}\right)}\right\}|\nabla \phi|^{2} \\
& +\left\{|\phi|^{2}-\frac{n(\mathrm{n}-2)}{\sqrt{\mathrm{n}(\mathrm{n}-1)}} \mathrm{H}|\phi|\right\}\left(\varepsilon+|\phi|^{2}\right) .
\end{aligned}
$$

Using (10) with $\boldsymbol{f}_{\varepsilon}$ as a test function,

$$
\begin{aligned}
\lambda_{1}^{\mathrm{L}_{H}} \int_{M}\left(\varepsilon+|\phi|^{2}\right) \mathrm{d} v & =\lambda_{1}^{\mathrm{L}_{H}} \int_{M} f_{\varepsilon}^{2} \mathrm{~d} v \leqslant \int_{M} \mathrm{f}_{\varepsilon} \mathrm{L}_{H}\left(\mathrm{f}_{\varepsilon}\right) \mathrm{d} v \\
& \leqslant-\int_{M}|\phi|^{2}\left\{|\phi|^{2}-\frac{\mathrm{n}(\mathrm{n}-2) \mathrm{H}}{\sqrt{\mathrm{n}(\mathrm{n}-1)}}|\phi|+\mathrm{n}\left(1-\mathrm{H}^{2}\right)\right\} \mathrm{d} v
\end{aligned}
$$




$$
\begin{aligned}
& -\int_{M}\left\{1-\frac{n|\phi|^{2}}{(n+2)\left(\varepsilon+|\phi|^{2}\right)}\right\}|\nabla \phi|^{2} \mathrm{~d} v \\
& +\int_{M}\left\{|\phi|^{2}-\frac{n(n-2)}{\sqrt{n(n-1)}} H|\phi|\right\}\left(\varepsilon+|\phi|^{2}\right) d v .
\end{aligned}
$$

Letting $\varepsilon \rightarrow \infty$ in (19),

$$
\lambda_{1}^{\mathrm{L}_{H}} \int_{M}|\phi|^{2} \mathrm{~d} v \leqslant-\mathrm{n}\left(1-\mathrm{H}^{2}\right) \int_{M}|\phi|^{2} \mathrm{~d} v-\int_{M} \frac{2}{\mathrm{n}+2}|\nabla \phi|^{2} \mathrm{~d} v .
$$

Since $\lambda_{1}^{\mathrm{L}_{\mathrm{H}}} \geqslant-\mathrm{n}\left(1-\mathrm{H}^{2}\right)$, from $(20),|\nabla \phi|^{2}=0$. Proposition 3 implies that $\nabla|\phi|^{2}=0$, that is, $|\phi|^{2}$ is constant. Therefore, we know that $|\phi|^{2}-$ $(n(n-2) / \sqrt{n(n-1)}) H|\phi|$ is constant. From (1), we obtain that $\lambda_{1}^{L_{H}}=$ $|\phi|^{2}-(n(n-2) / \sqrt{n(n-1)}) H|\phi|$. So

$$
-n\left(1-H^{2}\right) \leqslant|\phi|^{2}-\frac{n(n-2)}{\sqrt{n(n-1)}} H|\phi|,
$$

that is

$$
|\phi|^{2}-\frac{\mathrm{n}(\mathrm{n}-2)}{\sqrt{\mathrm{n}(\mathrm{n}-1)}} \mathrm{H}|\phi|+\mathrm{n}\left(1-\mathrm{H}^{2}\right) \geqslant 0 .
$$

Therefore, we know that the equalities in (12) and (13) hold and

$$
|\phi|^{2}\left\{|\phi|^{2}-\frac{n(n-2)}{\sqrt{n(n-1)}} H|\phi|+n\left(1-H^{2}\right)\right\}=0 .
$$

This implies that $|\phi|^{2}=0$, that is, $M$ is totally umbilical, or

$$
|\phi|^{2}-\frac{n(n-2)}{\sqrt{n(n-1)}} H|\phi|+n\left(1-H^{2}\right)=0 .
$$

In this case, the equalities hold in (13) and it follows that $M$ has at most two distinct constant principal curvatures. We conclude that $M$ is totally umbilical from the compactness of $M$. This completes the proof of Theorem 1 . 
Proof of 2: Since $R<1$, from the assertion in section 2 and the Gauss equation (7), we know that the operator $\square$ is elliptic and $n^{2} H^{2}=S+n(n-$ $1)(1-R)>0$. Hence, $H \neq 0$. Since $M$ is orientable, we can assume that $H>0$. Thus, from (11), we introduce a smooth function $f=H$ as the test function to estimate $\lambda_{1}^{L_{R}}$. By (2) and (14),

$$
\begin{aligned}
\mathrm{L}_{\mathrm{R}}(\mathrm{H})= & -\square(\mathrm{H})+\frac{1}{\mathrm{n}}|\phi|^{4}+\left(1-\mathrm{H}^{2}\right)|\phi|^{2} \\
\leqslant & -\left\{\frac{1}{\mathrm{n}}|\nabla \mathrm{h}|^{2}-\mathrm{n}|\nabla \mathrm{H}|^{2}+\frac{1}{\mathrm{n}}|\phi|^{4}+\left(1-\mathrm{H}^{2}\right)|\phi|^{2}-\frac{\mathrm{n}-2}{\sqrt{\mathrm{n}(\mathrm{n}-1)}} \mathrm{H}|\phi|^{3}\right\} \\
& +\frac{1}{\mathrm{n}}|\phi|^{4}+\left(1-\mathrm{H}^{2}\right)|\phi|^{2} \\
= & -\left(\frac{1}{\mathrm{n}}|\nabla \mathrm{h}|^{2}-\mathrm{n}|\nabla \mathrm{H}|^{2}\right)+\frac{\mathrm{n}-2}{\sqrt{\mathrm{n}(\mathrm{n}-1)}} \mathrm{H}|\phi|^{3} .
\end{aligned}
$$

From (11) and (15),

$$
\begin{aligned}
\lambda_{1}^{L_{R}} \int_{M} H^{2} \mathrm{~d} v & \leqslant \int_{M} H L_{R}(H) d v \\
& =-\int_{M} H\left(\frac{1}{n}|\nabla h|^{2}-n|\nabla H|^{2}\right) d v+\int_{M} \frac{n-2}{\sqrt{n(n-1)}} H^{2}|\phi|^{3} d v \\
& \leqslant \int_{M} \frac{n-2}{\sqrt{n(n-1)}} H^{2}|\phi|^{3} d v \leqslant \frac{n-2}{\sqrt{n(n-1)}} \max |\phi|^{3} \int_{M} H^{2} d v .
\end{aligned}
$$

Thus,

$$
\lambda_{1}^{L_{R}} \leqslant \frac{n-2}{\sqrt{n(n-1)}} \max |\phi|^{3} .
$$

If $\lambda_{1}^{L_{R}}=[(n-2) / \sqrt{n(n-1)}] \max |\phi|^{3}$, then the equalities in (22), (21), (15), (14) and (13) hold. Since the operator $\square$ is self-adjoint and $M$ is compact, from (14), we obtain that

$$
\int_{M}|\phi|^{2}\left\{|\phi|^{2}-\frac{n(n-2) H}{\sqrt{n(n-1)}}|\phi|+n\left(1-H^{2}\right)\right\} d v=0 .
$$


This implies that $|\phi|^{2}=0$ and $M$ is totally umbilical, or

$$
|\phi|^{2}-\frac{n(n-2) H}{\sqrt{n(n-1)}}|\phi|+n\left(1-H^{2}\right)=0 .
$$

By the Gauss equation (7), (23) is equivalent to

$$
\begin{aligned}
|\phi|^{2}- & \frac{n-2}{n-1}|\phi| \sqrt{|\phi|^{2}-n(n-1)(R-1)} \\
& +n\left(1-\frac{1}{n(n-1)}|\phi|^{2}+(R-1)\right)=0 .
\end{aligned}
$$

Since the scalar curvature $n(n-1) R$ is constant, from $(24),|\phi|$ is constant. By the equalities of (13), we know that $M$ has at most two distinct constant principal curvatures. We conclude that $M$ is totally umbilical from the compactness of $M$. This completes the proof of Theorem 2 .

Acknowledgement The project supported by NSF of Shaanxi Province (SJ08A31) and NSF of Shaanxi Educational Committee (2008JK484). The authors thank the referee for his/her many valuable suggestions that improved the paper.

\section{References}

[1] H. Alencar and M. do Carmo, Hypersurfaces with constant mean curvature in spheres, Pro. of the Amer. Math. Soc., 120(4) (1994), 1223-1229. http://www.jstor.org/pss/2160241 E88

[2] H. Alencar, M. do Carmo and A. G. Colares, Stable hypersurfaces with constant scalar curvature, Math. Z., 213 (1993), 117-131. doi:10.1007/BF03025712 E84 
[3] L. J. Alías, A. Barros, and A. Jr. Brasil, A spectral characterization of $\mathrm{H}(\mathrm{r})$-torus by the first stability eigenvalue, Proc. Amer. Math. Soc., 133 (2005), 875-884. doi:10.1090/S0002-9939-04-07559-8 E84, E85

[4] J. L. Barbosa, M. do Carmo and J. Eschenburg, Stability of hypersurfaces of constant mean curvature in Riemannian manifolds, Math. Z., 197 (1988), 123-138. doi:10.1007/BF01161634 E84

[5] J. L. Barbosa and V. Oliker, Stable space-like hypersurfaces with constant mean curvature in Lorentz space, Geometry and global Analysis, Tohoku Univ., Sendai, (1993), 161-164. E84, E85

[6] J. L. Barbosa and V. Oliker, Space-like hypersurfaces with constant mean curvature in Lorentz space, Mat. Contemp., 4 (1993), 27-44. E85

[7] A. Brasil. Jr, A. G. Colares and O. Palmas, Complete space-like hypersurfaces with constant mean curvature in the de Sitter space: A gap Theorem. Illinois Journal of Mathematics, 47 (2003), 847-866. http://projecteuclid.org/euclid.ijm/1258138197 E88

[8] A. A. Barros, A. C. Brasil Jr. and L. A. M. Sousa Jr., A new characterization of submanifolds with parallel mean curvature vector in $\mathrm{S}^{\mathrm{n}+\mathrm{p}}$, Kodai Math. J., 27 (2004), 45-56. doi:10.2996/kmj/1085143788 E89

[9] Q.-M. Cheng, First eigenvalue of a Jacobi operator of hypersurfaces with a constant scalar curvature, Proc. Amer. Math. Soc., 136 (2008), 3309-3318. doi:10.1090/S0002-9939-08-09304-0 E84, E85

[10] Q.-M. Cheng and S. Ishikawa, Space-like hypersurfaces in de Sitter spaces with constant scalar curvature, Manuscripta Math., 95 (1998), 499-505. doi:10.1007/BF02678045 E88

[11] S. Y. Cheng and S. T. Yau, Hypersurfaces with constant scalar curvature,Math. Ann., 225 (1977), 195-204. doi:10.1007/BF01425237 $\mathrm{E} 86, \mathrm{E} 88$ 
[12] X. Liu and J. Deng, Stable space-like hypersurfaces in the de Sitter space, Archivum Math. (Brno), 40(2004), 111-117.

http://dml.cz/dmlcz/107895 E84

[13] X. Liu, Complete space-like hypersurfaces with constant scalar curvature, Manuscripta Math., 105 (2001), 367-377. doi:10.1007/s002290100187 E89

[14] M. Okumura, Hypersurfaces and a pinching problem on the second fundamental tensor, Amer. J. Math., 96 (1974), 207-213. http://www.jstor.org/pss/2373587 E88

[15] S. C. Shu and S. Y. Liu, Complete space-like submanifolds with constant scalar curvature in a de Sitter space, Balkan J. of Geomety and its Appli., 9 (2004), 82-91. E89

[16] C. Wu, New characterizations of the Clifford tori and the Veronese surface, Arch. Math., 61 (1993), 277-284. doi:10.1007/BF01198725 $\mathrm{E} 84, \mathrm{E} 89$

\section{Author addresses}

1. Shichang Shu, Department of Mathematics, Xianyang Normal University, Xianyang 712000 Shaanxi, P.R. China mailto:shushichang@126.com

2. Sanyang Liu, Department of Applied Mathematics, Xidian University, Xi'an 710071 Shaanxi, P.R. China mailto:liusanyang@163.com 